\title{
Depathing maps for circulating state shifts
}

\author{
Mau-Hsiang Shih and Feng-Sheng Tsai* \\ Dedicated to Professor Wataru Takahashi on the occasion of his seventieth birthday.
}

\author{
"Correspondence: \\ fstsai@abel.math.ntnu.edu.tw \\ Department of Mathematics, \\ National Taiwan Normal University, \\ 88 Sec. 4, Ting Chou Road, Taipei, \\ 11677, Taiwan
}

\begin{abstract}
We have described a decirculation process which marks perturbations of network structure that are necessary for nonlinear network dynamics to proceed from one circulating state (a limit cycle) to another stable state (a limit cycle or a fixed point). Armed with the decirculation process, a sort of decirculating maps and their structural properties have also been built, dedicated to showing that circulation breaking taking place in nonlinear network dynamics can collaborate harmoniously toward the completion of network structure that generates attractors (equilibrium states). Here we wish to extend the notion of decirculating maps to the notion of depathing maps. The extension allows us to reshape network structure not only on the occasion of circulating states but on the occasion of any required path states. This gives a crucial improvement in generating circulating state shifts more feasibly. MSC: 47H10; 37F20; 92B20; 00A71; 68T05; 91E40
\end{abstract}

Keywords: fixed point; equilibrium; depathing maps; state shifts; nonlinear dynamics

\section{Introduction}

Pattern generation in complex biological systems may be understood by means of the concepts of nonlinear network dynamics [1,2]. The modeled systems can be formed by large numbers of interacting units whose dynamical properties tend to emerge through the collective interactions of many units. The modeled systems generally reach one of possible multiple stable states (alternative stable states) [3-5], which have multistability governed by the control parameters assigned to evolutionary network structure. State shifts between multiple stable states can be induced by the decirculation process [6], which marks a quantified determinant of the reshape of network structure that is sufficient for shifts from one circulating state (a limit cycle) to another stable state (a limit cycle or a fixed point). The decirculation process is generally stated as follows: 'the occurrence of a loop of unit states in the modeled systems leads to a change in network connections, which feeds back to reinforce interacting units to tend to break the circulation of unit states in this loop.'

Armed with the decirculation process, a sort of decirculating maps and their structural properties are built in $[7,8]$, dedicated to showing that circulation breaking taking place in nonlinear network dynamics can collaborate harmoniously toward the completion of network structure that generates attractors (equilibrium states). Here we wish to extend the notion of decirculating maps to the notion of depathing maps. The extension allows us to reshape network structure not only on the occasion of circulating states but on the occasion of any required path states. Hence it can generate circulating state shifts more

\section{空 Springer}

(c) 2013 Shih and Tsai; licensee Springer. This is an Open Access article distributed under the terms of the Creative Commons Attribution License (http://creativecommons.org/licenses/by/2.0), which permits unrestricted use, distribution, and reproduction in any medium, provided the original work is properly cited. 
feasibly. It reveals the depathing process which is generally stated as follows: 'the occurrence of a path of unit states in the modeled systems leads to a change in network connections, which feeds back to reinforce interacting units to tend to break the flow of unit states in this path.' Operator construction for path breaking is also put in the section at the end, displaying the tendency toward path breaking aiming to control nonlinear network dynamics.

\section{Depathing maps}

Let $\{0,1\}^{n}$ denote the binary code consisting of all 01-strings of fixed length $n$. Denote by $\Omega=\left[x^{0}, x^{1}, \ldots, x^{p}\right]$ a path of states in $\{0,1\}^{n}$, meaning that $p>1, x^{0}, x^{1}, \ldots, x^{p} \in\{0,1\}^{n}$, and $x^{0} \neq x^{i}$ for some $i \in\{1,2, \ldots, p\}$. Specifically, we call that $\Omega$ is a loop if $x^{0}=x^{p}$.

For every $i, j=1,2, \ldots, n$, we assign an integer, denoted by $c_{i j}(\Omega)$, according to the rule

$$
c_{i j}(\Omega)=x_{j}^{0}\left(x_{i}^{0}-x_{i}^{1}\right)+x_{j}^{1}\left(x_{i}^{1}-x_{i}^{2}\right)+\cdots+x_{j}^{p-1}\left(x_{i}^{p-1}-x_{i}^{p}\right) .
$$

We refer to the resulting matrix $C(\Omega)=\left(c_{i j}(\Omega)\right.$ ) as the depathing map of $\Omega$. (If $\Omega$ is a loop, then the depathing map $C(\Omega)$ is equivalent to the decirculating map defined in $[7,8]$, where we have explained why the terminology is used in connection with circulation breaking.) For example, let $\Omega=[1111100000,0011111000,0000111110,0111110000,0001111100]$. Then

$$
C(\Omega)=\left(\begin{array}{cccccccccc}
1 & 1 & 1 & 1 & 1 & 0 & 0 & 0 & 0 & 0 \\
1 & 2 & 2 & 2 & 1 & 0 & -1 & -1 & -1 & 0 \\
0 & 1 & 2 & 2 & 1 & 1 & 0 & -1 & -1 & 0 \\
0 & 0 & 1 & 1 & 0 & 0 & 0 & -1 & -1 & 0 \\
0 & 0 & 0 & 0 & 0 & 0 & 0 & 0 & 0 & 0 \\
-1 & -1 & -1 & -1 & -1 & 0 & 0 & 0 & 0 & 0 \\
-1 & -2 & -2 & -2 & -1 & 0 & 1 & 1 & 1 & 0 \\
0 & -1 & -2 & -2 & -1 & -1 & 0 & 1 & 1 & 0 \\
0 & 0 & -1 & -1 & 0 & 0 & 0 & 1 & 1 & 0 \\
0 & 0 & 0 & 0 & 0 & 0 & 0 & 0 & 0 & 0
\end{array}\right) .
$$

Consider the dynamical system of $n$ coupled units modeled by the equation $[6,9]$

$$
x(t+1)=H_{A}(x(t), s(t)), \quad t=0,1, \ldots,
$$

where $x(t)=\left(x_{1}(t), x_{2}(t), \ldots, x_{n}(t)\right) \in\{0,1\}^{n}$ is the vector of unit states at time $t, A=\left(a_{i j}\right) \in$ $M_{n}(\mathbb{R})$ is the coupling matrix of $n$ coupled units, $s(t) \subset\{1,2, \ldots, n\}$ denotes the units that adjust their states at time $t$, and $H_{A}(\cdot, s(t))$ is a function whose $i$ th component is defined by

$$
\left[H_{A}(x, s(t))\right]_{i}=\mathbb{1}\left(\sum_{j=1}^{n} a_{i j} x_{j}-b_{i}\right) \quad \text { if } i \in s(t),
$$

otherwise $\left[H_{A}(x, s(t))\right]_{i}=x_{i}$, where $b_{i} \in \mathbb{R}$ is the threshold of unit $i$ and the function $\mathbb{1}$ is the Heaviside function: $\mathbb{1}(u)=1$ for $u \geq 0$, otherwise 0 , which describes an instantaneous unit 
pulse. The dynamical system generates the vector of unit states according to (2), resulting in the phase flow $x(t), t=0,1, \ldots$.

With the depathing map $C(\Omega)$, we are bound to consider the linear functional $A \longrightarrow$ $\langle A, C(\Omega)\rangle$ on the Hilbert space $M_{n}(\mathbb{R})$ of all real $n \times n$ matrices endowed with the HilbertSchmidt inner product $\langle\cdot, \cdot\rangle$.

Theorem 2.1 Let $\Omega=\left[x^{0}, x^{1}, \ldots, x^{p}\right]$ be a path of states in $\{0,1\}^{n}$. If $A \in M_{n}(\mathbb{R})$ and $b \in \mathbb{R}^{n}$ satisfy

$$
\langle A, C(\Omega)\rangle \geq\left\langle b, x^{0}-x^{p}\right\rangle,
$$

then for any initial unit state $x(0) \in\{0,1\}^{n}$ and any updating $s(t) \subset\{1,2, \ldots, n\}, t=0,1, \ldots$, the resulting phase flow $x(t)$ of $(2)$ cannot behave in

$$
x(T)=x^{0}, x(T+1)=x^{1}, \ldots, x(T+p)=x^{p}
$$

for each $T=0,1, \ldots$

Proof For any 01-string $x=x_{1} x_{2} \cdots x_{n}$, we define

$$
\begin{aligned}
& \mathbf{1}(x)=\left\{i ; x_{i}=1,1 \leq i \leq n\right\}, \\
& \mathbf{0}(x)=\left\{i ; x_{i}=0,1 \leq i \leq n\right\} .
\end{aligned}
$$

Suppose, by contradiction, that there exist $b \in \mathbb{R}^{n}, x(0) \in\{0,1\}^{n}, s(t) \subset\{1,2, \ldots, n\}, t=$ $0,1, \ldots$, and $T \geq 0$ such that $x(T)=x^{0}, x(T+1)=x^{1}, \ldots, x(T+p)=x^{p}$. Let

$$
\begin{aligned}
& \Lambda^{+}=\{t ; \mathbf{0}(x(t)) \cap \mathbf{1}(x(t+1)) \neq \emptyset, T \leq t<T+p\}, \\
& \Lambda^{-}=\{t ; \mathbf{1}(x(t)) \cap \mathbf{0}(x(t+1)) \neq \emptyset, T \leq t<T+p\} .
\end{aligned}
$$

Then $\Lambda^{+} \neq \emptyset$ and $\Lambda^{-} \neq \emptyset$. Indeed, if $\Lambda^{+}=\emptyset$ or $\Lambda^{-}=\emptyset$, then

$$
x(T)=x(T+1)=\cdots=x(T+p)
$$

contradicting the path assumption $x(T) \neq x(T+p)$. According to (1), we have

$$
\begin{aligned}
\langle A, C(\Omega)\rangle & =\sum_{i, j} a_{i j}\left(\sum_{0 \leq m<p} x_{j}^{m} x_{i}^{m}-\sum_{0 \leq m<p} x_{j}^{m} x_{i}^{m+1}\right) \\
& =\sum_{0 \leq m<p}\left(\sum_{i, j} a_{i j} x_{j}^{m} x_{i}^{m}-\sum_{i, j} a_{i j} x_{j}^{m} x_{i}^{m+1}\right) \\
& =\sum_{0 \leq m<p}(\langle A x(T+m), x(T+m)\rangle-\langle A x(T+m), x(T+m+1)\rangle) \\
& =\sum_{0 \leq m<p}\langle A x(T+m), x(T+m)-x(T+m+1)\rangle .
\end{aligned}
$$


Since $\mathbf{0}(x(t)) \cap \mathbf{1}(x(t+1)) \subset s(t)$ and $\mathbf{1}(x(t)) \cap \mathbf{0}(x(t+1)) \subset s(t)$ for each $t=0,1, \ldots$, we conclude from (2) that

$$
\begin{aligned}
& \sum_{0 \leq m<p}\langle A x(T+m), x(T+m)-x(T+m+1)\rangle \\
& <-\sum_{t \in \Lambda^{+}} \sum_{j \in \mathbf{0}(x(t)) \cap \cap \mathbf{1}(x(t+1))} b_{j}+\sum_{t \in \Lambda^{-}} \sum_{j \in \mathbf{1}(x(t)) \cap \cap \mathbf{0}(x(t+1))} b_{j} \\
& =\sum_{0 \leq m<p}\langle b, x(T+m)-x(T+m+1)\rangle \\
& =\langle b, x(T)-x(T+p)\rangle .
\end{aligned}
$$

Combining (4) and (5) shows that $\langle A, C(\Omega)\rangle<\left\langle b, x^{0}-x^{p}\right\rangle$, contradicting (3), and that completes the proof.

\section{Operator control on path breaking}

Denote by $\Omega=\left[x^{0}, x^{1}, \ldots, x^{p}\right]$ a path of states in $\{0,1\}^{n}$. For each $m=0,1, \ldots, p$, we say that the state $x^{m}$ is in the position $m$ of the path $\Omega$. For each 01-string $x=x_{1} x_{2} \cdots x_{n}$, let

$$
\begin{aligned}
& \mathbf{1}(x)=\left\{i ; x_{i}=1,1 \leq i \leq n\right\} \\
& \mathbf{0}(x)=\left\{i ; x_{i}=0,1 \leq i \leq n\right\} .
\end{aligned}
$$

Let us recall that the symmetric difference of two sets $U$ and $V$ is the set $U \Delta V$, each of whose elements belongs to $U$ but not to $V$, or belongs to $V$ but not to $U$. For every $i=1,2, \ldots, n$, let

$$
\begin{aligned}
& M_{i}(\Omega)=\left\{m ; i \in \mathbf{1}\left(x^{m-1}\right) \triangle \mathbf{1}\left(x^{m}\right), m=1,2, \ldots, p\right\}, \\
& M_{i}(\Omega)^{+}=\left\{m ; i \in \mathbf{1}\left(x^{m}\right) \backslash \mathbf{1}\left(x^{m-1}\right), m=1,2, \ldots, p\right\}, \\
& M_{i}(\Omega)^{-}=\left\{m ; i \in \mathbf{1}\left(x^{m-1}\right) \backslash \mathbf{1}\left(x^{m}\right), m=1,2, \ldots, p\right\} .
\end{aligned}
$$

Here $M_{i}(\Omega)$ denotes the collection of the positions $m$ of the path $\Omega$, in which unit $i$ changes its state from $x_{i}^{m-1}=0$ to $x_{i}^{m}=1$ or from $x_{i}^{m-1}=1$ to $x_{i}^{m}=0$, whereas $M_{i}(\Omega)^{+}\left(\operatorname{resp} ., M_{i}(\Omega)^{-}\right)$ denotes the collection of the positions $m$ of the path $\Omega$, in which unit $i$ changes its state from $x_{i}^{m-1}=0$ to $x_{i}^{m}=1$ (resp., changes its state from $x_{i}^{m-1}=1$ to $x_{i}^{m}=0$ ). For every $i, j=$ $1,2, \ldots, n$, define

$$
\begin{aligned}
\Upsilon_{i j}(\Omega)= & \sharp\left(M_{i}(\Omega)^{+} \cap M_{j}(\Omega)^{+}\right)+\sharp\left(M_{i}(\Omega)^{-} \cap M_{j}(\Omega)^{-}\right) \\
& -\sharp\left(M_{i}(\Omega)^{+} \cap M_{j}(\Omega)^{-}\right)-\sharp\left(M_{i}(\Omega)^{-} \cap M_{j}(\Omega)^{+}\right),
\end{aligned}
$$

which can be regarded as a measure of synchronous activity between units $i, j$, that is, if units $i, j$ tend to change their states synchronously (resp., asynchronously) in $\Omega$, then $\Upsilon_{i j}(\Omega)>0\left(\right.$ resp., $\left.\Upsilon_{i j}(\Omega)<0\right)$. For every $i, j=1,2, \ldots, n$, define

$$
\Gamma_{i j}(\Omega)=\min \left\{\sharp M_{i}(\Omega), \sharp M_{j}(\Omega)\right\},
$$


which can be regarded as a measure of self-sustaining activity of units $i, j$, that is, if unit $i$ or $j$ tends to maintain more self-sustaining states in $\Omega$ than unit $i^{\prime}$ or $j^{\prime}$, then $\Gamma_{i j}(\Omega)<\Gamma_{i^{\prime} j^{\prime}}(\Omega)$. We refer to the resulting matrices $\Upsilon(\Omega)=\left(\Upsilon_{i j}(\Omega)\right)$ and $\Gamma(\Omega)=\left(\Gamma_{i j}(\Omega)\right)$ as the measure of synchronous activity and the measure of self-sustaining activity derived from the path $\Omega$ of states in $\{0,1\}^{n}$, respectively.

Let $\bar{\Omega}=\left[x^{0}, x^{1}, \ldots, x^{p}, x^{0}\right]$. Denote by $\left.\langle\cdot, \cdot\rangle\right\rangle$ the Hilbert-Schmidt inner product in $M_{n}(\mathbb{R})$, i.e., if $A=\left(a_{i j}\right)$ and $B=\left(b_{i j}\right) \in M_{n}(\mathbb{R})$, then $\langle A, B\rangle=\operatorname{tr}\left(A B^{T}\right)=\sum_{i, j} a_{i j} b_{i j}$. Define $I(\Omega)=$ $\mathbf{1}\left(x^{p}\right) \cap \mathbf{1}\left(x^{0}\right)$. Let

$$
\begin{aligned}
\mathcal{D}(\Omega)= & \left\{\mathcal{D}_{\mathrm{SY}}+\mathcal{D}_{\mathrm{SK}} ; \mathcal{D}_{\mathrm{SY}}=\mathcal{D}_{\mathrm{SY}}^{T} \in M_{n}(\mathbb{R}), \mathcal{D}_{\mathrm{SK}}=-\mathcal{D}_{\mathrm{SK}}^{T} \in M_{n}(\mathbb{R})\right. \text { with } \\
& \left(\mathcal{D}_{\mathrm{SK}}\right)_{i j} \geq 0 \text { for each }(i, j) \in\left(\mathbf{1}\left(x^{0}\right) \times \mathbf{1}\left(x^{p}\right)\right) \backslash(I(\Omega) \times I(\Omega)), \text { and } \\
& \left.\left\langle\left|\mathcal{D}_{\mathrm{SK}}\right|, \Gamma(\bar{\Omega})\right\rangle<\left\langle\mathcal{D}_{\mathrm{SY}}, \Upsilon(\bar{\Omega})-C\left(\left[x^{p}, x^{0}\right]\right)\right\rangle\right\},
\end{aligned}
$$

where $\left|\mathcal{D}_{\mathrm{SK}}\right|=\left(\left|\left(\mathcal{D}_{\mathrm{SK}}\right)_{i j}\right|\right)$. The set $\mathcal{D}(\Omega)$ collects all the combining operations of the operators $\mathcal{D}_{\mathrm{SY}}$ and $\mathcal{D}_{\mathrm{SK}}$, which will determine a clamp of network modification by $A+\mathcal{D}_{\mathrm{SY}}+\mathcal{D}_{\mathrm{SK}}$.

Fix $\mathcal{D}_{\mathrm{SY}}+\mathcal{D}_{\mathrm{SK}} \in \mathcal{D}(\Omega)$. Define $I_{1}(\Omega)=\mathbf{1}\left(x^{p}\right) \cap \mathbf{0}\left(x^{0}\right)$ and $I_{2}(\Omega)=\mathbf{0}\left(x^{p}\right) \cap \mathbf{1}\left(x^{0}\right)$. Since $I_{1}(\Omega) \cap I(\Omega)=\emptyset, I_{1}(\Omega) \cup I(\Omega)=\mathbf{1}\left(x^{p}\right), I_{2}(\Omega) \cap I(\Omega)=\emptyset$, and $I_{2}(\Omega) \cup I(\Omega)=\mathbf{1}\left(x^{0}\right)$, we have

$$
\left(\mathbf{1}\left(x^{0}\right) \times \mathbf{1}\left(x^{p}\right)\right) \backslash(I(\Omega) \times I(\Omega))=\left(I(\Omega) \times I_{1}(\Omega)\right) \cup\left(I_{2}(\Omega) \times \mathbf{1}(x(p))\right),
$$

and hence, by (9),

$$
\begin{aligned}
\left\langle\mathcal{D}_{\mathrm{SK}}, C\left(\left[x^{p}, x^{0}\right]\right)\right\rangle & =\sum_{(i, j) \in I_{1}(\Omega) \times \mathbf{1}(x(p))}\left(\mathcal{D}_{\mathrm{SK}}\right)_{i j}-\sum_{(i, j) \in I_{2}(\Omega) \times \mathbf{1}(x(p))}\left(\mathcal{D}_{\mathrm{SK}}\right)_{i j} \\
& =\sum_{(i, j) \in I_{1}(\Omega) \times I_{1}(\Omega)}\left(\mathcal{D}_{\mathrm{SK}}\right)_{i j}+\sum_{(i, j) \in I_{1}(\Omega) \times I(\Omega)}\left(\mathcal{D}_{\mathrm{SK}}\right)_{i j}-\sum_{(i, j) \in I_{2}(\Omega) \times \mathbf{1}(x(p))}\left(\mathcal{D}_{\mathrm{SK}}\right)_{i j} \\
& \leq 0 .
\end{aligned}
$$

Furthermore, according to the proof in [8, Theorem 1], the following assertion holds:

$$
\left\langle\mathcal{D}_{\mathrm{SY}}+\mathcal{D}_{\mathrm{SK}}, C(\bar{\Omega})\right| \geq\left\langle\mathcal{D}_{\mathrm{SY}}, \Upsilon(\bar{\Omega})\right\rangle-\left\langle\left|\mathcal{D}_{\mathrm{SK}}\right|, \Gamma(\bar{\Omega})\right\rangle
$$

Combining (9), (10), and (11) shows that

$$
\begin{aligned}
\left\langle\mathcal{D}_{\mathrm{SY}}+\mathcal{D}_{\mathrm{SK}}, C(\Omega)\right\rangle= & \left\langle\mathcal{D}_{\mathrm{SY}}+\mathcal{D}_{\mathrm{SK}}, C(\bar{\Omega})-C\left(\left[x^{p}, x^{0}\right]\right)\right\rangle \\
\geq & \left\langle\mathcal{D}_{\mathrm{SY}}, \Upsilon(\bar{\Omega})\right\rangle-\left\langle\left|\mathcal{D}_{\mathrm{SK}}\right|, \Gamma(\bar{\Omega})\right\rangle \\
& -\left\langle\mathcal{D}_{\mathrm{SY}}, C\left(\left[x^{p}, x^{0}\right]\right)\right\rangle-\left\langle\mathcal{D}_{\mathrm{SK}}, C\left(\left[x^{p}, x^{0}\right]\right)\right\rangle \\
> & 0 .
\end{aligned}
$$

With the notation and arguments above, we describe operator control on path breaking as follows.

Theorem 3.1 Let $A \in M_{n}(\mathbb{R})$ and $b \in \mathbb{R}^{n}$. Let $\Omega=\left[x^{0}, x^{1}, \ldots, x^{p}\right]$ be a path of states in $\{0,1\}^{n}$. Then, for every operator $\mathcal{D}_{\mathrm{SY}}+\mathcal{D}_{\mathrm{SK}} \in \mathcal{D}(\Omega)$, there exists $\gamma \geq 0$ such that

$$
\left\langle A+\gamma\left(\mathcal{D}_{\mathrm{SY}}+\mathcal{D}_{\mathrm{SK}}\right), C(\Omega)\right\rangle \geq\left\langle b, x^{0}-x^{p}\right\rangle .
$$


Hence, by Theorem 2.1, the dynamical system of $n$ coupled units modeled by the equation

$$
x(t+1)=H_{A+\gamma\left(\mathcal{D}_{\mathrm{SY}}+\mathcal{D}_{\mathrm{SK}}\right)}(x(t), s(t)), \quad t=0,1, \ldots,
$$

cannot behave in

$$
x(T)=x^{0}, x(T+1)=x^{1}, \ldots, x(T+p)=x^{p}
$$

for each $T=0,1, \ldots$

In the following, we give an example to construct a sort of operators $\mathcal{D}_{\mathrm{SY}}+\mathcal{D}_{\mathrm{SK}} \in \mathcal{D}(\Omega)$. Let $n=10$ and $p=4$. Let $\Omega=\left[x^{0}, x^{1}, x^{2}, x^{3}, x^{4}\right]=[1111100000,0011111000,0000111110$, 0111110000, 0001111100]. Then

$$
\begin{aligned}
& I(\Omega)=\mathbf{1}\left(x^{p}\right) \cap \mathbf{1}\left(x^{0}\right)=\{4,5\}, \\
& I_{1}(\Omega)=\mathbf{1}\left(x^{p}\right) \cap \mathbf{0}\left(x^{0}\right)=\{6,7,8\}, \\
& I_{2}(\Omega)=\mathbf{0}\left(x^{p}\right) \cap \mathbf{1}\left(x^{0}\right)=\{1,2,3\} .
\end{aligned}
$$

Associate to each $i \in\{1,2, \ldots, n\}$ a real number $\varepsilon_{i}$ such that

(1) for each $i, j \in\{1,2, \ldots, n\}$, if $\left(\sum_{m} x_{i}^{m}\right) /\left(M_{i}(\Omega)+1\right)>\left(\sum_{m} x_{j}^{m}\right) /\left(M_{j}(\Omega)+1\right)$, then

$$
\varepsilon_{i} \leq \varepsilon_{j}
$$

(2) $\sum_{1 \leq i \leq n} M_{i}(\Omega) \varepsilon_{i}+\sum_{i \in I_{2}(\Omega)} \varepsilon_{i}>0$

(see Table 1 for a choice of $\left.\varepsilon_{i}\right)$. We may select $y=\left(y_{1}, y_{2}, \ldots, y_{n}\right) \in \mathbb{R}^{n}$ and $\mathcal{D}_{\mathrm{SY}}=\left(y_{i} y_{j}+\delta_{i j} \varepsilon_{i}\right) \in$ $M_{n}(\mathbb{R})$, where $\delta_{i j}=1$ if $i=j$, otherwise 0 , such that exactly one of the following holds:

(1) $y_{i} \geq 0$ for $i \in \mathbf{1}\left(x^{p}\right)$ and $\sum_{i \in I_{1}(\Omega)} y_{i} \leq \sum_{i \in I_{2}(\Omega)} y_{i}$;

(2) $y_{i} \leq 0$ for $i \in \mathbf{1}\left(x^{p}\right)$ and $\sum_{i \in I_{1}(\Omega)} y_{i} \geq \sum_{i \in I_{2}(\Omega)} y_{i}$

(see Table 1 for a choice of $y_{i}$ and Table 2 for $\mathcal{D}_{\mathrm{SY}}$ ). Consider the shift function $\sigma$ on $\{0,1, \ldots, p\}$ given by

$$
\sigma(k) \equiv k+1 \quad \bmod p+1
$$

for each $k=0,1, \ldots, p$. Since $\left(y_{i} y_{j}\right) \in M_{n}(\mathbb{R})$ is positive semidefinite, we have

$$
\begin{aligned}
\left\langle\mathcal{D}_{\mathrm{SY}}, \Upsilon(\bar{\Omega})-C\left(\left[x^{p}, x^{0}\right]\right)\right\rangle= & \sum_{0 \leq m \leq p}\left\langle\mathcal{D}_{\mathrm{SY}}\left(x^{m}-x^{\sigma(m)}\right),\left(x^{m}-x^{\sigma(m)}\right)\right\rangle \\
& -\sum_{j \in \mathbf{1}\left(x^{p}\right)} y_{j}\left(\sum_{i \in I_{1}(\Omega)} y_{i}-\sum_{i \in I_{2}(\Omega)} y_{i}\right)-\sum_{i \in I_{1}(\Omega)} \varepsilon_{i} \\
\geq & \sum_{0 \leq m<p} \sum_{i \in \mathbf{1}\left(x^{m}\right) \Delta \mathbf{1}\left(x^{\sigma(m)}\right)} \varepsilon_{i}+\sum_{i \in I_{2}(\Omega)} \varepsilon_{i} \\
= & \sum_{1 \leq i \leq n} M_{i}(\Omega) \varepsilon_{i}+\sum_{i \in I_{2}(\Omega)} \varepsilon_{i}>0 .
\end{aligned}
$$

For such a choice of $\mathcal{D}_{\mathrm{SY}}$, let $\varepsilon>0$ be such that

$$
\gamma_{\Omega}=\left(\left\langle\mathcal{D}_{\mathrm{SY}}, \Upsilon(\bar{\Omega})-C\left(\left[x^{p}, x^{0}\right]\right)\right\rangle-\varepsilon\right) /\|\Gamma(\bar{\Omega})\| \geq 0,
$$


Table 1 Operator construction: choose $\varepsilon_{i}$ and $y_{i}$

\begin{tabular}{lllllllllll}
\hline$\Omega=[1111100000,0011111000,0000111110,0111110000,0001111100]$ \\
$i$ & 1 & 2 & 3 & 4 & 5 & 6 & 7 & 8 & 9 & 10 \\
$M_{i}(\Omega)$ & 1 & 3 & 3 & 2 & 0 & 1 & 3 & 3 & 2 & 0 \\
$\sum_{m} x_{i}^{m}$ & 1 & 2 & 3 & 4 & 5 & 4 & 3 & 2 & 1 & 0 \\
$\left(\sum_{m} x_{i}^{m}\right) /\left(M_{i}(\Omega)+1\right)$ & $1 / 2$ & $1 / 2$ & $3 / 4$ & $4 / 3$ & 5 & 2 & $3 / 4$ & $1 / 2$ & $1 / 3$ & 0 \\
$\varepsilon_{i}$ & 0.8 & 0.5 & 0.5 & -0.7 & -1.8 & -1 & -0.3 & 0.5 & 1.2 & 1.5 \\
$y_{i}$ & 3 & 1 & -1 & 0 & 0.5 & 1 & 1 & 1 & 0 & -1 \\
\hline
\end{tabular}

Table 2 Operator construction: construct the operator $\mathcal{D}_{\mathrm{SY}}=\left(y_{i} y_{j}+\delta_{i j} \varepsilon_{i}\right)$

\begin{tabular}{cccccccccc}
\hline 9.8 & 3 & -3 & 0 & 1.5 & 3 & 3 & 3 & 0 & -3 \\
3 & 1.5 & -1 & 0 & 0.5 & 1 & 1 & 1 & 0 & -1 \\
-3 & -1 & 1.5 & 0 & -0.5 & -1 & -1 & -1 & 0 & 1 \\
0 & 0 & 0 & -0.7 & 0 & 0 & 0 & 0 & 0 & 0 \\
1.5 & 0.5 & -0.5 & 0 & -1.55 & 0.5 & 0.5 & 0.5 & 0 & -0.5 \\
3 & 1 & -1 & 0 & 0.5 & 0 & 1 & 1 & 0 & -1 \\
3 & 1 & -1 & 0 & 0.5 & 1 & 0.7 & 1 & 0 & -1 \\
3 & 1 & -1 & 0 & 0.5 & 1 & 1 & 1.5 & 0 & -1 \\
0 & 0 & 0 & 0 & 0 & 0 & 0 & 0 & 1.2 & 0 \\
-3 & -1 & 1 & 0 & -0.5 & -1 & -1 & -1 & 0 & 2.5 \\
\hline
\end{tabular}

Table 3 Operator construction: construct the operator $\mathcal{D}_{\mathrm{SK}}=\alpha S$ satisfying (12) and (13)

\begin{tabular}{lllllllllc}
\hline 0 & 0 & -0.015 & 0 & 0.005 & 0 & 0.02 & 0.01 & -0.015 & -0.01 \\
0 & 0 & 0.01 & 0 & 0.015 & 0.01 & 0.015 & 0.015 & 0.01 & -0.005 \\
0.015 & -0.01 & 0 & 0.015 & 0 & 0.01 & 0.01 & 0.01 & 0.005 & 0.01 \\
0 & 0 & -0.015 & 0 & -0.005 & 0.005 & 0.01 & 0.01 & 0 & -0.01 \\
-0.005 & -0.015 & 0 & 0.005 & 0 & 0.005 & 0.01 & 0.005 & 0.015 & 0 \\
0 & -0.01 & -0.01 & -0.005 & -0.005 & 0 & -0.01 & 0.015 & 0.01 & -0.02 \\
-0.02 & -0.015 & -0.01 & -0.01 & -0.01 & 0.01 & 0 & 0.005 & 0.015 & 0.005 \\
-0.01 & -0.015 & -0.01 & -0.01 & -0.005 & -0.015 & -0.005 & 0 & 0.005 & 0.015 \\
0.015 & -0.01 & -0.005 & 0 & -0.015 & -0.01 & -0.015 & -0.005 & 0 & 0 \\
0.01 & 0.005 & -0.01 & 0.01 & 0 & 0.02 & -0.005 & -0.015 & 0 & 0 \\
\hline
\end{tabular}

Table 4 Operator construction: construct the combining operation $\mathcal{D}_{\mathrm{SY}}+\mathcal{D}_{\mathrm{SK}}$

\begin{tabular}{llllllllll}
\hline 9.8 & 3 & -3.015 & 0 & 1.505 & 3 & 3.02 & 3.01 & -0.015 & -3.01 \\
3 & 1.5 & -0.99 & 0 & 0.515 & 1.01 & 1.015 & 1.015 & 0.01 & -1.005 \\
-2.985 & -1.01 & 1.5 & 0.015 & -0.5 & -0.99 & -0.99 & -0.99 & 0.005 & 1.01 \\
0 & 0 & -0.015 & -0.7 & -0.005 & 0.005 & 0.01 & 0.01 & 0 & -0.01 \\
1.495 & 0.485 & -0.5 & 0.005 & -1.55 & 0.505 & 0.51 & 0.505 & 0.015 & -0.5 \\
3 & 0.99 & -1.01 & -0.005 & 0.495 & 0 & 0.99 & 1.015 & 0.01 & -1.02 \\
2.98 & 0.985 & -1.01 & -0.01 & 0.49 & 1.01 & 0.7 & 1.005 & 0.015 & -0.995 \\
2.99 & 0.985 & -1.01 & -0.01 & 0.495 & 0.985 & 0.995 & 1.5 & 0.005 & -0.985 \\
0.015 & -0.01 & -0.005 & 0 & -0.015 & -0.01 & -0.015 & -0.005 & 1.2 & 0 \\
-2.99 & -0.995 & 0.99 & 0.01 & -0.5 & -0.98 & -1.005 & -1.015 & 0 & 2.5 \\
\hline
\end{tabular}

where $\|\Gamma(\bar{\Omega})\|=\langle\Gamma(\bar{\Omega}), \Gamma(\bar{\Omega})\rangle^{\frac{1}{2}}$. Then, for any choice of $S \in M_{n}(\mathbb{R})$ with $S=-S^{T}$ and $S_{i j} \geq 0$ for each $(i, j) \in\left(\mathbf{1}\left(x^{0}\right) \times \mathbf{1}\left(x^{p}\right)\right) \backslash(I(\Omega) \times I(\Omega))$, we set $\mathcal{D}_{\mathrm{SK}}=\alpha S$, where $\alpha \in \mathbb{R}$ is such that

$$
\left\|\mathcal{D}_{\mathrm{SK}}\right\|=\left\langle\mathcal{D}_{\mathrm{SK}}, \mathcal{D}_{\mathrm{SK}}\right\rangle^{\frac{1}{2}}=\alpha\langle S, S\rangle^{\frac{1}{2}} \leq \gamma_{\Omega}
$$

(see Table 3 for a choice of $\mathcal{D}_{\mathrm{SK}}$ ). Thus, by (12) and (13), we have

$$
\left\langle\left|\mathcal{D}_{\mathrm{SK}}\right|, \Gamma(\bar{\Omega})\right\rangle<\left\langle\mathcal{D}_{\mathrm{SY}}, \Upsilon(\bar{\Omega})-C\left(\left[x^{p}, x^{0}\right]\right)\right\rangle .
$$

Hence $\mathcal{D}_{\mathrm{SY}}+\mathcal{D}_{\mathrm{SK}} \in \mathcal{D}(\Omega)$ (see Table 4 for a choice of $\left.\mathcal{D}_{\mathrm{SY}}+\mathcal{D}_{\mathrm{SK}}\right)$ 
Competing interests

The authors declare that they have no competing interests.

\section{Authors' contributions}

Both authors contributed equally and significantly in writing this paper. Both authors read and approved the final manuscript.

\section{Acknowledgements}

This work was supported by the National Science Council of Taiwan.

Received: 17 June 2013 Accepted: 4 July 2013 Published: 22 July 2013

\section{References}

1. Schöner, G, Kelso, JAS: Dynamic pattern generation in behavioral and neural systems. Science 239, 1513-1520 (1988)

2. Strogatz, SH: Nonlinear Dynamics and Chaos: With Applications to Physics, Biology, Chemistry, and Engineering. Westview Press, Cambridge (2000)

3. May, RM: Thresholds and breakpoints in ecosystems with a multiplicity of stable states. Nature 269, 471-477 (1977)

4. Beisner, BE, Haydon, DT, Cuddington, K: Alternative stable states in ecology. Front. Ecol. Environ. 1, 376-382 (2003)

5. Scheffer, M, Carpenter, S, Foley, JA, Folke, C, Walker, B: Catastrophic shifts in ecosystems. Nature 413, $591-596$ (2001)

6. Shih, MH, Tsai, FS: Decirculation process in neural network dynamics. IEEE Trans. Neural Netw. Learn. Syst. 23(11), 1677-1689 (2012)

7. Shih, MH, Tsai, FS: Neural network dynamics without minimizing energy. Abstr. Appl. Anal. 2013, Article ID 496217 (2013)

8. Shih, MH, Tsai, FS: Operator control of interneural computing machines. IEEE Trans. Neural Netw. Learn. Syst. (2013). doi:10.1109/TNNLS.2013.2271258

9. Shih, MH, Tsai, FS: Growth dynamics of cell assemblies. SIAM J. Appl. Math. 69, 1110-1161 (2009)

doi:10.1186/1687-1812-2013-195

Cite this article as: Shih and Tsai: Depathing maps for circulating state shifts. Fixed Point Theory and Applications 2013 2013:195.

\section{Submit your manuscript to a SpringerOpen ${ }^{\ominus}$ journal and benefit from:}

- Convenient online submission

- Rigorous peer review

- Immediate publication on acceptance

- Open access: articles freely available online

- High visibility within the field

- Retaining the copyright to your article 